\title{
The optical to $\gamma$-ray emission of the Crab pulsar: a multicomponent model
}

\author{
E. Massaro ${ }^{1}$, R. Campana ${ }^{1}$, G. Cusumano ${ }^{2}$, and T. Mineo ${ }^{2}$ \\ 1 Dipartimento di Fisica, Università di Roma “La Sapienza”, Piazzale A. Moro 2, 00185, Roma, Italy \\ e-mail: enrico.massaro@uniroma1.it \\ 2 INAF-IASF, Sezione di Palermo, via Ugo La Malfa 153, 90146, Palermo, Italy \\ Received 1 March 2006 / Accepted 20 June 2006
}

ABSTRACT

\begin{abstract}
We present a multicomponent model to explain the features of the pulsed emission and spectrum of the Crab Pulsar, on the basis of $\mathrm{X}$ and $\gamma$-ray observations obtained with BeppoSAX, INTEGRAL and CGRO. This model explains the evolution of the pulse shape and of the phase-resolved spectra, ranging from the optical/UV to the GeV energy band, on the assumption that the observed emission is due to more components. The first component, $C_{O}$, is assumed to have the pulsed double-peaked profile observed at the optical frequencies, while the second component, $C_{X}$, is dominant in the interpeak and second peak phase regions. The spectra of these components are modelled with log-parabolic laws and their spectral energy distributions have peak energies at 12.2 and $178 \mathrm{keV}$, respectively. To explain the properties of the pulsed emission in the $\mathrm{MeV}-\mathrm{GeV}$ band, we introduce two more components, $C_{O \gamma}$ and $C_{X \gamma}$, with phase distributions similar to those of $C_{O}$ and $C_{X}$ and log-parabolic spectra with the same curvature but peak energies at about $300 \mathrm{MeV}$ and $2 \mathrm{GeV}$. This multicomponent model is able to reproduce both the broadband phase-resolved spectral behaviour and the changes of the pulse shape with energy. We also propose some possible physical interpretations in which $C_{O}$ and $C_{X}$ are emitted by secondary pairs via a synchrotron mechanism while $C_{O \gamma}$ and $C_{X \gamma}$ can originate either from Compton scattered or primary curvature photons.
\end{abstract}

Key words. stars: pulsars: individual: crab pulsar (PSR B0531+21) - X-rays: stars - gamma rays: observations stars: pulsars: general

\section{Introduction}

The origin of the high energy emission of rotation-powered pulsars is still an unsolved problem. One of the main difficulties is related to the description of the phase and energy distributions of the pulsed signal, which depends on both a physical and geometrical modelling of the magnetosphere. It is still unclear, for example, whether electrons (or positrons) are accelerated and radiate streaming out the polar cap regions (Ruderman \& Sutherland 1975; Salvati \& Massaro 1978; Sturner \& Dermer 1994; Daugherty \& Harding 1994, 1996; Muslimov \& Harding 2003) or in the outer gaps (Cheng et al. 1986a,b; Chiang \& Romani 1994; Romani \& Yadigaroglu 1995; Cheng et al. 2000; Zhang \& Cheng 2002; Hirotani et al. 2003). Another important problem concerns how quantum processes, like magnetic pair production (Erber 1966) and photon splitting (Adler 1971), modify the high energy $\gamma$-ray spectrum.

The Crab pulsar (PSR B0531+21) has been the best studied object of this class since its discovery (Staelin \& Reifenstein 1968) and the amount of data collected is rich enough to search for a detailed physical picture of its emission properties. It is well known that the pulse shape of Crab has a characteristic double peak structure, with a phase separation of 0.4 , detected from the radio band to $\gamma$ rays and changing with energy. A very remarkable feature is that the so-called second peak, hereafter P2, in the $\mathrm{X}$ and soft $\gamma$-ray ranges becomes progressively higher than the first peak (P1). A similar increase is also evident in the emission between the peaks, usually named the Interpeak region (Ip) or bridge (see Fig. 1). Above about $10 \mathrm{MeV}, \mathrm{P} 1$ is again the dominant feature. A satisfactory explanation for these changes has not been found so far.
On the basis of high quality BeppoSAX data, covering a wide energy range from 0.1 to about $300 \mathrm{keV}$, we proposed a two component model (Massaro et al. 2000, hereafter MCLM) to interpret this behaviour. In the same paper we studied in detail the energy spectrum of the core of P1 (corresponding to a phase interval having a width of only 0.027 around the maximum) which shows a continuous steepening at high energies. We found that in the energy range $0.1-300 \mathrm{keV}$ this spectral distribution is well represented by a parabolic law in a double logarithmic plot (hereafter log-parabola) with a rather mild curvature. The extrapolation of this model in the $\gamma$-ray range, however, fails to reproduce the data and a more complex modelling is required.

In a subsequent paper Kuiper et al. (2001) introduced three components to describe the spectrum up to the $\gamma$-ray data obtained with COMPTEL and EGRET on board ComptonGRO. These authors based the analysis mainly on finding best fits of the spectral distributions in rather narrow phase intervals and found that the spectral variation of the pulsed emission with phase can be modelled by two log-parabolic components with a relative normalization changing with phase. A further third power law component, having a photon index equal to 2.07, was necessary for the emission of P1 and P2 to to match the EGRET data at energies higher than about $10 \mathrm{MeV}$.

In this paper we develop a model able to describe the phase and spectral distributions of the emission over a frequency interval from the optical frequencies to $\mathrm{GeV}$ range. This model is an extension of that presented in MCLM and it based on the results of a new detailed data analysis of many BeppoSAX observations which includes PDS data from March 1999 to April 2001 not considered by MCLM. The timing accuracy has been verified 
Table 1. Log of BeppoSAX NFIs Crab Pulsar pointing epochs and net exposure times.

\begin{tabular}{crrrr}
\hline \hline \multicolumn{2}{c}{ Observation } & \multicolumn{4}{c}{ Exposure Times (s) $^{\text {Date }}$} & LECS & MECS $^{a}$ & HPGSPC & \multicolumn{1}{c}{ PDS } \\
\hline 31 Aug. 1996 & - & - & 28384 & - \\
6 Sep. 1996 & 5733 & 33482 & - & - \\
30 Sep. 1996 & - & 7874 & - & 3596 \\
12 Apr. 1997 & 1257 & 18551 & 9344 & 9338 \\
8 Oct. 1997 & 12096 & 30769 & 9158 & 19673 \\
6 Apr. 1998 & 8730 & 28694 & 12732 & - \\
13 Oct. 1998 & - & - & - & 17476 \\
9 Mar. 1999 & - & - & - & 13674 \\
25 Sep. 1999 & - & - & - & 15417 \\
10 Apr. 2000 & - & - & - & 15602 \\
4 Apr. 2001 & - & - & - & 7597 \\
\hline Total exposure & 27816 & 119370 & 59618 & 102373 \\
\hline
\end{tabular}

${ }^{a}$ MECS operated with two detectors after May 1997.

using detailed pulse profiles obtained from RXTE archive data. Moreover, to extend the energy range, we analysed several more recent observations performed with the IBIS-ISGRI experiment on board the INTEGRAL satellite and considered the results of Kuiper et al. (2001) on the COMPTEL and EGRET observations up to a few $\mathrm{GeV}$. The main goal of our work is the definiton of a scenario that can be used to develop more detailed physical models of the Crab pulsar high-energy emission.

\section{Observations and data reduction}

\subsection{BeppoSAX}

BeppoSAX observed the Crab Nebula and its pulsar on several occasions during its lifetime, because this very bright source was used to monitor the responses of the four Narrow Field Instruments (NFI). All the data collected up to 1998 were used in MCLM and a complete description of their reduction is presented in that work. In the new analysis presented in this paper we did not consider some early PDS observations, particularly those performed on 31 August 1996, during the Science Verification Phase, and on 6 April 1998 because the detector gain was found to be not precisely set, whereas additional PDS data were obtained from other observations performed between 1999 and 2001, corresponding to an increase of the exposure time of about $12 \%$ with respect to MCLM. The complete log of all BeppoSAX pointings used in the present work is given in Table 1.

For the imaging instruments we selected all the events within circular regions centered on the source position and having radii of $4^{\prime}$ (MECS) and $8^{\prime}$ (LECS). This choice corresponds to using about $90 \%$ of the total source signal in both instruments, but it allows us to apply the best tested spectral response matrices.

Phase histograms of the Crab pulsar were evaluated for each NFI and each pointing using the period folding technique. The UTC arrival times of all selected events were converted to the Solar System Barycentre with the DE200 ephemeris. The values of $P$ and $\dot{P}$ for each observation epoch were derived from the Jodrell Bank Crab Pulsar Monthly Ephemeris ${ }^{1}$. We constructed a large set of 300 bin phase histograms for each energy channel of each NFI. The zero phase was fixed at the centre of the first peak, evaluated by Gaussian fits. All the histograms for the same

\footnotetext{
${ }^{1}$ http://www.jb.man.ac.uk/
}

energy channel of each NFI were then added. Before this operation we verified that all the profiles of the various observation epochs had fully compatible shapes and therefore similar folding accuracy. A summary of all these phase histograms is shown in the upper panels of Fig. 1.

A relevant point we stress when working with spectra obtained with several instruments is that a proper evaluation of the inter-calibration factors is required. For the BeppoSAX NFIs, the accurate ground and in-flight calibrations were used to establish the admissible ranges for the factors between the MECS and the other three instruments: $k_{\mathrm{ML}}$ for the LECS, $k_{\mathrm{MH}}$ for the HPGSPC and $k_{\text {MP }}$ for the PDS (Fiore et al. 1999). When performing the spectral analysis, we left these three parameters free and found the values $k_{\mathrm{ML}}=0.74$ and $k_{\mathrm{MH}}=0.88$, within the ranges given by Fiore et al. (1999). For point sources these authors gave $0.77 \leq k_{\mathrm{MP}} \leq 0.93$, reduced to $0.86 \pm 0.03$ for sources with a PDS count rate higher than $2 \mathrm{ct} / \mathrm{s}$. We found in our best fits $k_{\mathrm{MP}}=0.82$, very close to the above prescription, and that we considered fully satisfactory because it was derived with the logparabolic model instead of the simple power law used by Fiore et al. (1999).

\subsection{RossiXTE}

To verify the accuracy of BeppoSAX timing, we compared the pulse profiles as observed by MECS with that obtained by the PCA instrument (Jahoda et al. 1996) on board of the Rossi $\mathrm{X}$-Ray Timing Explorer. The latter instrument has a timing accuracy of $2 \mu$ s (Rots et al. 2004) with respect to the spacecraft clock and an absolute time accuracy of 5-8 $\mu$ s with respect to UTC. We used the RXTE observation of Crab performed on December 3, 2004. PCA data were obtained in an "event mode" that provided 128 energy channels with $250 \mu$ s time resolution. PCA data were screened following standard selection criteria excluding time intervals corresponding to South Atlantic Anomaly passages, Earth limb closer than 10 degrees and with angular distance between the source and pointing direction larger than 0.02 . The total exposure time after all screening criteria was $709 \mathrm{~s}$. After the conversion of the arrival times to the Solar System Barycentre by using the DE200 ephemeris, phase histograms were obtained by folding data with the Jodrell Bank Crab Pulsar Monthly Ephemeris as before.

Figure 2 shows the RossiXTE-PCA pulse profile, extracted in the $2.6-4.1 \mathrm{keV}$ energy band and with 300 phase bins $(110 \mu \mathrm{s})$, together with that obtained in the same energy range with MECS. Both profiles have been normalized to unity at the maximum of $\mathrm{P} 1$, after subtraction of the off-pulse constant level (0.6-0.83) and arbitrarily shifted to set the zero phase at the centre of the first peak. The comparison shows that the peak widths between the two instruments are very similar. The two profiles have differences of only a few percent in amplitude (Fig. 2, lower panel) and there is no evidence of a systematic effect on the timing in the MECS data. We conclude that the BeppoSAX profiles accumulated over multiple observations do not undergo any significant broadening due either to the clock time assignment or phase misalignment among the different histograms added together.

\subsection{INTEGRAL}

To gain more information at energies higher than $200 \mathrm{keV}$ we considered also the recent data obtained with IBIS-ISGRI (Lebrun et al. 2003) on board the INTEGRAL satellite. In our 
Table 2. Log of INTEGRAL-ISGRI observations of the Crab and of net exposure times.

\begin{tabular}{cr}
\hline \hline Start - Stop day & Exposure Times (s) \\
\hline 07 Feb. 2003 - 09 Feb. 2003 & 142300 \\
10 Feb. 2003 - 11 Feb. 2003 & 56000 \\
16 Feb. 2003 - 18 Feb. 2003 & 61600 \\
19 Feb. 2003 - 21 Feb. 2003 & 30700 \\
22 Feb. 2003 - 24 Feb. 2003 & 9800 \\
25 Feb. 2003 - 26 Feb. 2003 & 2100 \\
15 Aug. 2003 - 17 Aug. 2003 & 37900 \\
17 Aug. 2003 - 17 Aug. 2003 & 21600 \\
\hline Total exposure & 362000 \\
\hline
\end{tabular}

analysis we considered only the IBIS observations having an offaxis angle not larger than $1^{\circ}$ which are listed in Table 2. Phase histograms with 100 bins were obtained for each pointing using, as before, the period folding technique (see Fig. 1). The accuracy of INTEGRAL timing with respect to RXTE has been verified by Kuiper et al. (2003) The zero phase was shifted at the centre of the first peak, coherently with the assumption for the other instruments. A more complete spectral analysis of these data, together with those obtained using other INTEGRAL instruments, can be found in Mineo et al. (2006).

\section{The interstellar absorption in the Crab direction}

\subsection{X-ray absorption}

An important piece of information for the study of the intrinsic shape of the soft X-ray spectral distribution is the value of the equivalent hydrogen column density and the composition of the interstellar matter in the Crab direction. In MCLM we assumed $N_{\mathrm{H}}=3.2 \times 10^{21} \mathrm{~cm}^{-2}$ and a mean solar composition. This value is higher than those considered in previous papers, usually lower than $3 \times 10^{21} \mathrm{~cm}^{-2}$ (see, for instance, Toor \& Seward 1977; Ride \& Walker 1977; Pravdo \& Serlemitsos 1981) and in agreement with the analysis of ASCA data by Fukuzawa et al. (1997) and with the result of Sollerman et al. (2000) who estimated $N_{\mathrm{H}}=3.2 \pm 0.5 \times 10^{21} \mathrm{~cm}^{-2}$ from STIS/HST observations of the Ly $\alpha$ line. This column density, however, was not confirmed by subsequent studies that indicated an even larger $N_{\mathrm{H}}$ and different chemical abundances. Willingale et al. (2001) on the basis of EPIC-MOS data found a column density of $3.45 \times 10^{21} \mathrm{~cm}^{-2}$ with an underabundance of oxygen and iron of $63 \%$ with respect to the solar composition. Weisskopf et al. (2004) obtained $N_{\mathrm{H}}=4.2 \times 10^{21} \mathrm{~cm}^{-2}$ with the abundances given by Wilms et al. (2000) and using a photoelectric absorption model that includes the scattering due to interstellar grains.

In order to use a more precise estimate of $N_{\mathrm{H}}$ and the most suited chemical composition we performed a new analysis of the X-ray spectrum of the DC emission of Crab (pulse phases 0.60-0.83) using LECS and MECS data combined. Background, estimated from archive blank fields, was subtracted from the events. We adopted a simple power law $\left(F(E)=K\left(E / E_{0}\right)^{-\Gamma}\right)$ as the best fitting model because in this band there is no evidence of a change of the nebular spectral index with energy (see next Section). The considered abundances and photoelectric cross sections were those of the models aneb+wabs (Anders \& Ebihara 1982; Morrison \& McCammon 1983) and angr $+\mathrm{bcmc}$ (Anders \& Grevesse 1989; Balucinska-Church \& McCammon 1992, vphabs) all implemented in the XSPEC package. The results are reported in Table 3, where for each model we give the best fit values of $N_{\mathrm{H}}$ and of the photon index $\Gamma$, the fraction of oxygen and iron with respect to the default value and the reduced $\chi^{2}$. All the fits were acceptable (reduced $\chi^{2}<1$ ) and the column densities were systematically higher than $3.2 \times$ $10^{21} \mathrm{~cm}^{-2}$. Photon index values were found to be very stable with a mean value of 2.124. We also found (see Sect. 6.2) that the residuals at energies lower than $0.8 \mathrm{keV}$ had a systematic deviation which disappeared using column density values around $3.6 \times 10^{21} \mathrm{~cm}^{-2}$. We decided, therefore, to adopt in our analysis this $N_{\mathrm{H}}$ value together with the angr+bcmc model, as indicated by other instruments with higher spectral capabilities.

\subsection{Optical-UV extinction}

The high estimate of the equivalent column density and the possible difference of the chemical composition with respect to the solar one could also affect the optical-UV extinction in the direction of Crab. The analysis of Sollerman et al. (2000) converges around a value of $E(B-V)=0.50$ mag and indicates that the extinction curve closely follows the standard one. Thus the $N_{\mathrm{H}} / E(B-V)$ ratio is equal to $6.9 \times 10^{21} \mathrm{~cm}^{-2} \mathrm{mag}^{-1}$, about $30 \%$ higher than the value reported by Predehl \& Schmitt (1995).

Such a relevant optical-UV intrinsic extinction makes the evaluation of the pulsed flux and spectrum quite difficult at these frequencies. Several authors (e.g. Percival et al. 1993; Sollerman et al. 2000) agree that the dereddened spectrum is a power law with a spectral index close to zero. The difference to uncorrected data is quite large: in fact, the observed spectral index is around -2 , and small differences in the values of $E(B-V)$ or $A_{\mathrm{V}}$ can modify the dereddened slope and flux. Of course, the extrapolation of UV data in the X-ray range may be affected by large uncertainties.

\section{Phase resolved X-ray spectroscopy}

In MCLM we showed that a simple power law does not provide an acceptable representation of the spectral distribution of the central bins of $\mathrm{P} 1$, corresponding to the narrow phase interval $0.99-0.01667$. This is not a specific property of $\mathrm{P} 1$ but can be extended to the entire pulsed signal as apparent from the phase resolved spectroscopy of Fig. 1, where pulse profiles and best fit photon indices evaluated in the energy bands of the four NFIs of BeppoSAX and of ISGRI-INTEGRAL are shown. Upper panels show the pulse profiles while lower panels show the phase evolution of the photon indices: note the well known increase of Ip and P2 with respect to P1 and that photon indices are also increasing with energy: that of P1 changes from about 1.6 to 2.4 and that of the Ip from 1.3 to 1.95 . The power law fit was applied to the events after the subtraction of a DC level, estimated from the phase interval $0.60-0.83$, and assumed to be representative of the nebular continuum. High resolution X-ray images obtained with Chandra (Tennant et al. 2001) showed that there is a weak emission from the pulsar also in this phase interval. Its mean intensity is of the order of few percent than that of the peaks and becomes negligible compared to the nebular emission when considering data extracted from wider angular beams, as in our case.

To study the behaviour of the photon index with energy in detail we considered the following three phase intervals representative of P1 (0.9833-0.0167), the Interpeak (Ip) region (0.14-0.25) and P2 (0.3833-0.4137). This choice was made to have intervals wide enough to obtain estimates of $\Gamma$ with small statistical uncertainties and sufficiently narrow to consider the emission of P1 mainly due to one of the components introduced by MCLM. 
Table 3. Estimates of the equivalent hydrogen column density $N_{\mathrm{H}}$, from combined LECS $(0.1-4 \mathrm{keV})$ and MECS (1.6-10 keV) observations with various absorption models and chemical abundances.

\begin{tabular}{cccccc}
\hline \hline Model & $N_{\mathrm{H}}\left(^{*}\right)$ & $\Gamma$ & $F_{O}$ & $F_{F e}$ & $\chi_{r}^{2}$ \\
\hline aneb+wabs & $3.54 \pm 0.01$ & $2.124 \pm 0.001$ & - & - & 0.89 \\
angr+bcmc & $3.33 \pm 0.01$ & $2.124 \pm 0.001$ & - & - & 0.99 \\
angr+bcmc & $3.62 \pm 0.04$ & $2.125 \pm 0.001$ & $0.81 \pm 0.02$ & - & 0.90 \\
angr+bcmc & $3.63 \pm 0.04$ & $2.124 \pm 0.001$ & $0.83 \pm 0.03$ & $0.87 \pm 0.05$ & 0.88 \\
angr+bcmc & $3.42 \pm 0.02$ & $2.123 \pm 0.001$ & - & $0.74 \pm 0.04$ & 0.93 \\
\hline
\end{tabular}

$\left.{ }^{*}\right)$ In units of $10^{21} \mathrm{~cm}^{-2}$.

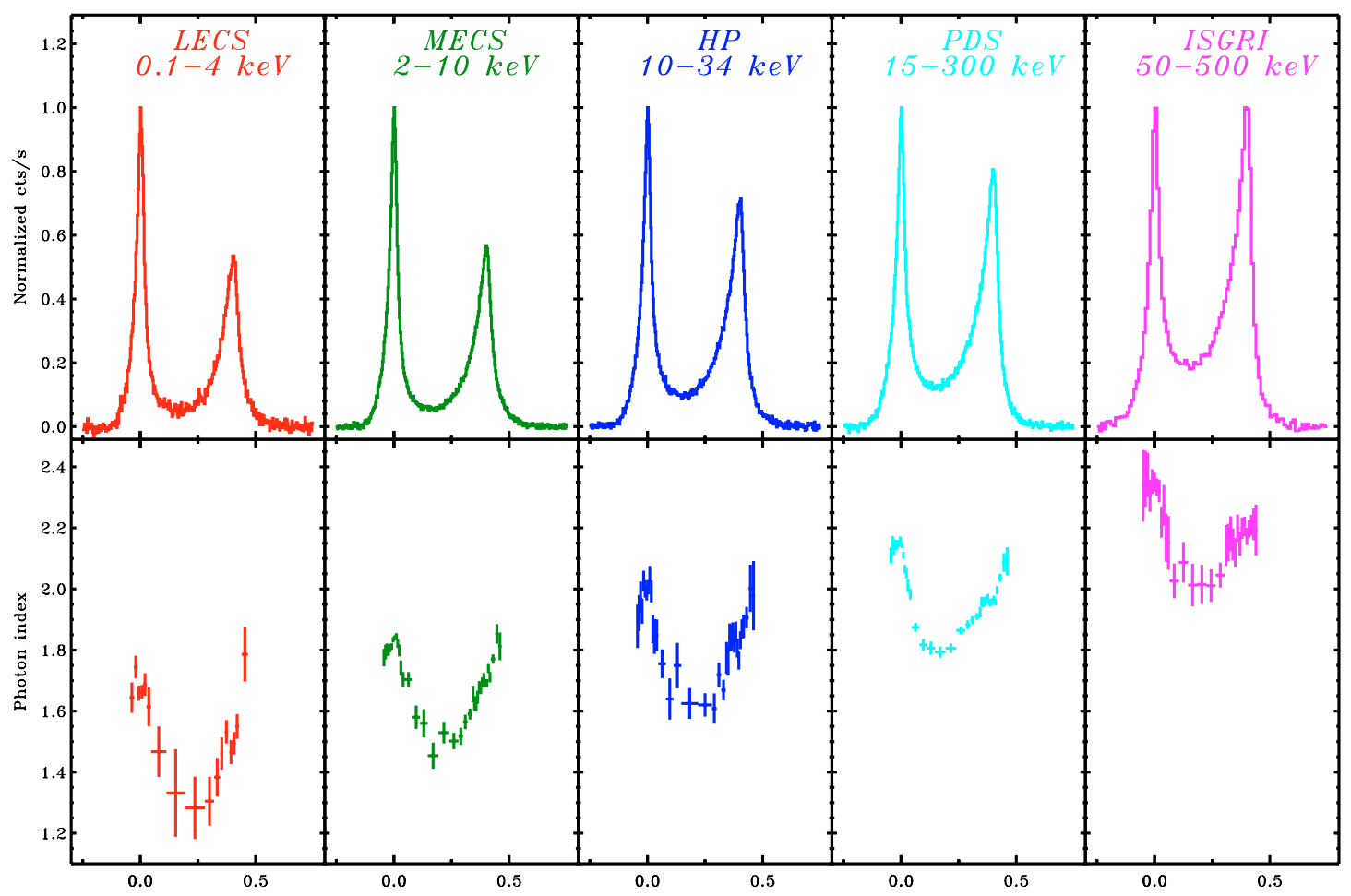

Pulse Phase

Fig. 1. The pulse profile and the phase dependent photon index of the Crab pulsar observed with the four Narrow Field Instruments of BeppoSAX and with ISGRI-INTEGRAL experiment. The respective energy ranges are indicated in the upper panels. All the profiles are normalized to unity at the maximum of P1. Note the change of relative intensity of P2 and Ip with respect to P1 and the increase of the photon indices.

In subsequent analysis, when comparing the results of our multicomponent model with literature data (see Sect. 7), it was necessary to consider for P1 and P2 phase intervals broader than those given above and we adopted the same definition given by Kuiper et al. (2001). The phase interval of Ip was the same. Figure 2 shows the differences between these two sets.

The resulting values of $\Gamma$ in the narrow phase intervals are shown in Fig. 3, together with those of the off-pulse, assumed originating in the nebula. The values of the P1 photon index vary from $1.72 \pm 0.02$ in the $0.1-4 \mathrm{keV}$ range to $2.30 \pm 0.05$ at (80-200 keV); correspondingly, those of Ip vary from $1.31 \pm 0.10$ to $1.98 \pm 0.10$ and those of P2 from $1.51 \pm 0.03$ to $2.14 \pm 0.05$. We considered also INTEGRAL-ISGRI data in the rather broad interval 50-500 keV and obtained photon indices fully consistent with the increasing trend of BeppoSAX values. The nebular spectrum has a remarkably constant value equal to $2.131 \pm 0.002$, up to the PDS range where it slightly increases to $2.172 \pm 0.006$ $(15-80 \mathrm{keV})$ and to $2.23 \pm 0.02(80-200 \mathrm{keV})$. Note that the last value agrees very well with that obtained by Kuiper et al. (2001) from COMPTEL data between 0.75 and $30 \mathrm{MeV}$. The very good consistency of the nebular photon index measured in LECS, MECS and HPGSPC is an indication of the high accuracy of their calibration and of the correct choice of the intercalibration constants.

It is clear from these results that a law more complex than a simple power law must be used to describe the broad-band spectral properties of the Crab pulsar when the data of all the NFIs are considered. A simple power law must be rejected because of the high values of the reduced $\chi^{2}$, found always larger than 2 .

In the MCLM analysis of the P1 spectrum, good spectral fits are obtained using a second order law in the double log representation:

$F(E)=A\left(E / E_{0}\right)^{-\left(a+b \log \left(E / E_{0}\right)\right)}$,

where $E_{0}$ is taken equal to $1 \mathrm{keV}$, and therefore $a$ corresponds to the photon index at this energy, while $b$ measures the curvature of the spectral distribution. We can also define an energy dependent photon index:

$\Gamma(E)=-\frac{\mathrm{d} \log F(E)}{\mathrm{d} \log E}=a+2 b \log \left(E / E_{0}\right)$. 


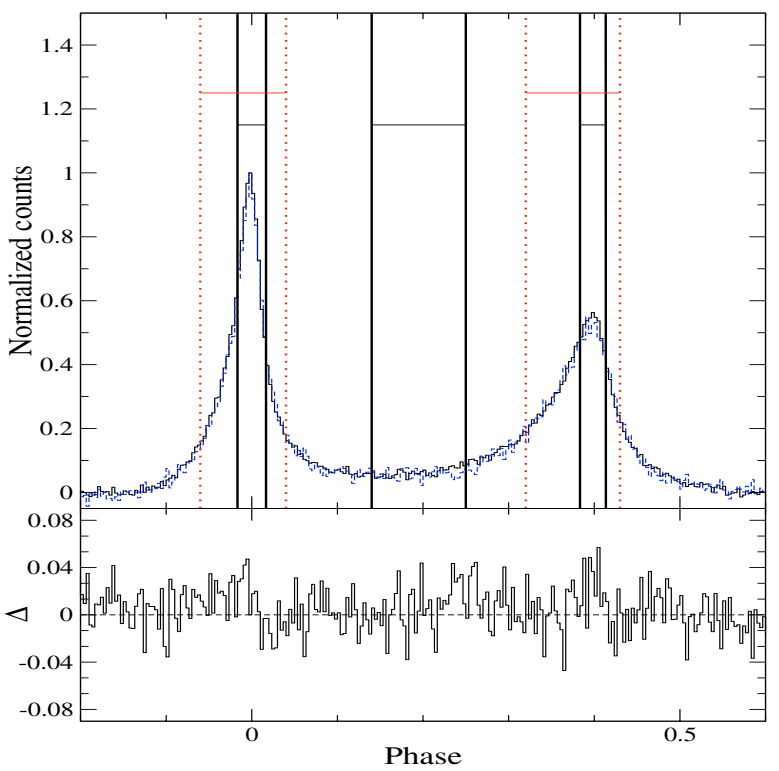

Fig. 2. The comparison of the normalized pulse profiles in the 2.6-4.1 keV energy range of BeppoSAX-MECS (solid histogram) and RossiXTE-PCA (dashed histogram) (upper panel). The difference plot in the lower panel has an amplitude of only a few percent and no sign of systematic effects on the timing. In the upper panel we also show the phase intervals of P1, Ip and P2 used in our spectral analysis (solid vertical lines - black) and according to Kuiper et al. (2001) (dotted vertical lines - red) used to compute the broad-band spectral distributions and the P2/P1 and Ip/P1 ratios (see Sect. 4).

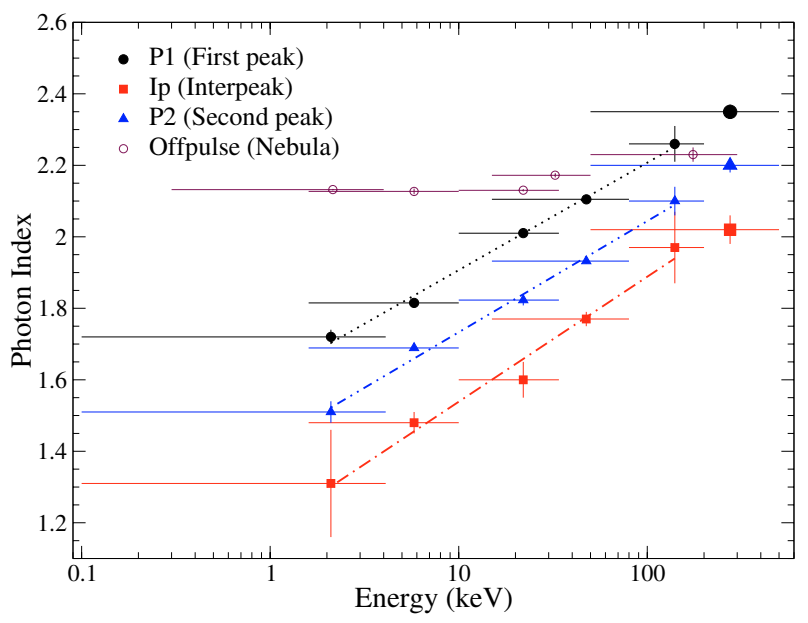

Fig. 3. The energy dependence of the photon indices of P1, Ip and P2, evaluated in the ranges of the LECS, MECS , HPGSPC, two PDS bands (15-80, 80-200 keV) and INTEGRAL-ISGRI (50-500 keV, large symbols). The off-pulse photon index, representative of the nebular emission, is also shown. Best-fit linear interpolations of BeppoSAX photon indices of P1, Ip and P2 are plotted.

This law implies then a linear relation between $\Gamma$ and $\log E$ like that apparent for the best fit lines plotted in Fig. 3, which have remarkably similar slopes corresponding to a curvature parameter $b$ between 0.13 and 0.16 , the former corresponding to $\mathrm{P} 2$ and the latter to Ip.

We are also interested in the spectral energy distribution, defined as $E^{2} F(E)$, which for a $\log$-parabolic law has a maximum at the energy:

$E_{\mathrm{p}}=10^{(2-a) / 2 b}$.

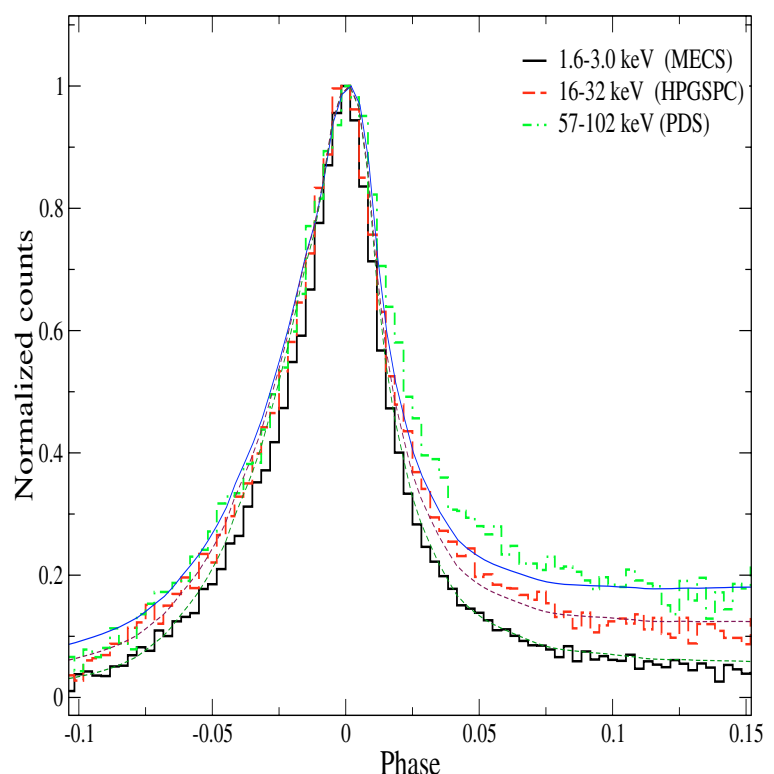

Fig. 4. The shape of P1 in three energy ranges. Maxima have been normalized to unity for a better comparison. Note the broadening of the peak with a marked asymmetry on the right side. Solid lines represent the P1 profiles derived using the two component model (see Sect. 6.3).

Mineo et al. (2006) used a log-parabola for the phase resolved spectroscopy of the INTEGRAL observations of Crab and found a significant curvature which was found stable across the entire phase interval of the pulsed signal with a mean value of $0.14 \pm 0.02$. We apply this law to each of the two components introduced by MCLM, as described in detail in Sect. 6.2.

\section{Shape and spectrum of the first peak}

Figure 4 shows the normalized profiles of P1 in the three energy bands $1.6-3 \mathrm{keV}, 16-32 \mathrm{keV}$ and $87-300 \mathrm{keV}$. As noticed by Mineo et al. (1997), there is an evident broadening which increases with energy. In the lowest energy range the peak profile is asymmetric with a leading side higher than the trailing one, as observed at optical frequencies (see, e.g., Kanbach et al. 2003). This asymmetry changes in the hard X-rays as the trailing side becomes progressively higher. The broadening, however, can be safely detected also on the leading side. We verified that this effect is also present to the same extent in the RXTE data and therefore it cannot be due to possible phase misalignements of the various BeppoSAX data sets.

Pravdo et al. (1997), using a RXTE observation of the Crab pulsar, found that the central part of P1 has a spectrum softer than the wings. In MCLM we confirmed this result from MECS data, but it was after questioned by Vivekanand (2002), who in a new analysis of the RXTE data in the range 5-60 keV obtained a stable value of $\Gamma$ across P1. We performed a detailed analysis with a finer phase resolution and confirmed the previous results: the photon index in the MECS range changes from $\sim 1.7$ in the wings of P1 to 1.82 in the core, as shown in the plot of Fig. 5. The same effect is also apparent in the PDS data. As it will be clearer in the following section this result is relevant to explain the total pulsed emission as due to the superposition of two components with different spectra. 


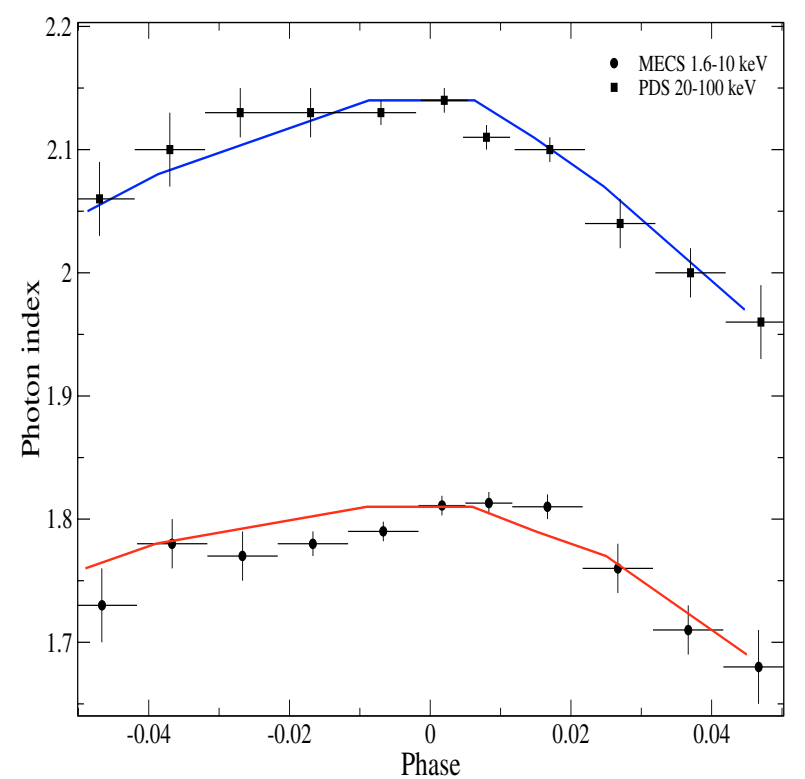

Fig. 5. Fine phase resolved photon index across $\mathrm{P} 1$ in the $1.6-10 \mathrm{keV}$ (MECS) and 20-100 keV (PDS) energy ranges. Solid lines are the photon indices derived using the two-component model as explained in Sect. 6.4

\section{The two component model}

To explain at the same time the change of the pulse profile and the phase evolution of the photon index, in MCLM we proposed a simple model in which the observed pulsed emission is considered as the superposition of two components having different phase distributions and energy spectra. The first component was assumed to have the same pulsed profile observed at optical frequencies (Smith et al. 1988), with P1 much more prominent than P2 and a very low intensity in the Ip region. The reason for this choice is that a similar shape is also observed at energies higher than $30 \mathrm{MeV}$, despite the profile of $\mathrm{P} 2$ being slightly different (Fierro et al. 1998). This component was called "optical" ( shortly $C_{O}$ ). The other "X-ray" component $C_{X}$ reaches the greatest relative intensity in the hard $X$ to low-energy $\gamma$-rays and it is necessary to explain the change of the $\mathrm{P} 2 / \mathrm{P} 1$ ratio. We derived its phase profile to reproduce the observed one when it is added to $C_{O}$ : we found that $C_{X}$ increases monotonically up to the phase 0.4 and then it has a rather sharp cut-off (Fig. 6). The main properties of the latter component were then estimated from the BeppoSAX data, with a fitting procedure of several pulse profiles at different energies, as explained in detail in MCLM.

In this new analysis we followed the same approach and considered again the same two components with only slight changes, as explained in the following subsections.

\subsection{The components' shapes}

We obtained the phase profile of $C_{O}$ from the recent light curve observed with the high speed photo-polarimeter OPTIMA with a time binning of $112 \mu$ s (Kanbach et al. 2003), comparable to that used for the BeppoSAX profiles. We used a high resolution digital scanner to convert the OPTIMA data and then we applied a rebinning to have the final phase profile with 300 bins, the same used for the X-ray data. This profile is shown in the upper panel of Fig. 6.

The pulse shape of $C_{X}$ can be obtained by the difference between the observed profiles and $C_{O}$. However, using the same approach as in MCLM we preferred to model $C_{X}$ by means of an analytical expression to make simpler computations. $C_{X}$ was factorized in two functions:

$F_{X}(E, f)=Y(E) g(f, E)$,

where $Y(E)$ is assumed to depend only on the photon energy $E$ and represents the spectral distribution of the component to be derived from the data as in MCLM, while $g(f, E)$ describes the phase behaviour and depends on both variables. In MCLM we found that the assumption that $g$ is only a function of the phase was not fully satisfied and a moderate change of the phase profile with the energy will be considered in this paper for a more precise description of the data. The whole phase interval of $C_{X}$ is denoted by $\left(f_{1}, f_{4}\right)$ and it is divided by two inner points at the phases $f_{2}$ and $f_{3}$ into three segments: in $\left(f_{1}, f_{2}\right)$ the $C_{X}$ shape is given by a power law, in $\left(f_{2}, f_{3}\right)$ by an exponential function joining the first one at $f_{2}$, and finally a linearly descending branch from $f_{3}$ to $f_{4}$ that connects the maximum to the zero level of the off-pulse. We have then:

$g(f, E)=\exp \left\{p(E)\left(f_{2}-f_{3}\right)\right\}\left(\frac{f-f_{1}}{f_{2}-f_{1}}\right)^{s}$,

for $f_{1}<f<f_{2}$, and

$g(f, E)=\exp \left\{p(E)\left(f-f_{3}\right)\right\}$,

for $f_{2}<f<f_{3}$.

We emphasize that $C_{X}$ is non zero also in the phase interval of P1: this choice is necessary to explain the broadening of this peak with increasing energy and the softer spectrum of the peak centre with respect to both wings (see Sect. 5.1). Note, however, that because of the dominance of $\mathrm{P} 1$ with respect to Ip in $C_{O}$, is it difficult to establish the actual shape of $C_{X}$ across P1. Our assumption that it has the smooth power law shape of Eq. (5) must be considered as one of the simplest approximations.

In MCLM we found that the phase interval boundaries, equal to $f_{1}=-0.15, f_{2}=0.21, f_{3}=0.40, f_{4}=0.43$ and the power law exponent $s=0.4$ can be considered constant with the energy, whereas a better agreement with the data is obtained if $p$ is allowed to vary, changing from about 11 in the $\mathrm{keV}$ range to 8 above $150 \mathrm{keV}$. A simple regression gives the relation:

$p(E)=-1.5811 \ln (\log E)+9.318$

that we used in our calculations. An example of how these two components combine to reproduce the observed profile in the energy range 8-10 keV is shown in Fig. 6.

Even though this model has some minor defects of accuracy, it gives an acceptable picture of the broad-band spectral and phase properties of the pulsed emission of Crab, as we will show in the following sections. This model is not only a useful tool to represent a limited data set and it can be successfully used to extrapolate the pulse shape and spectra outside the energy range in which it is established. Note that Kuiper et al. (2001) derived a seven-bin phase profile of their component necessary to explain the spectral difference between Ip and the peaks and found a structure similar to $C_{X}$.

\subsection{Spectral properties of the two components}

To model the phase and spectral properties of the total emission as the superposition of $C_{O}$ and $C_{X}$ we had to estimate their spectral distributions. The low intensity of $C_{O}$ in the Ip interval can be used to represent its X-ray flux as due only to the $C_{X}$, 


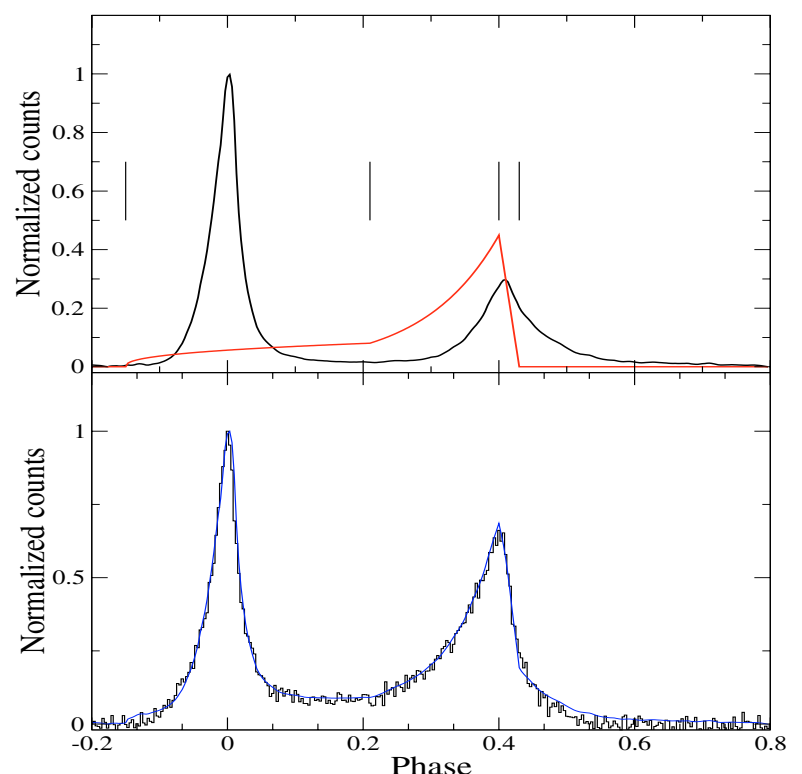

Fig. 6. Upper panel: phase distributions of the components $C_{O}$ and $C_{X}$ with the proper normalizations. $C_{O}$ is derived from OPTIMA lightcurve (Kanbach et al. 2003). The four vertical bars indicate the phases from $f_{1}$ to $f_{4}$ used in the analytical model of $C_{X}$. Lower panel: comparison between the observed pulse profile in the energy interval $(8.0-10.0) \mathrm{keV}$ (MECS data) and that obtained using the two component model computed for the mean energy of $8.85 \mathrm{keV}$.

while in the first and second peaks both components contribute to the total emission, although with different relative intensities. Assuming that the spectrum of each component is given by a log-parabolic law, we have for P1, Ip and P2:

$F(E)_{\mathrm{P} 1}=\left(A_{O}\right)_{\mathrm{P} 1} E^{-\left(a_{O}+b_{O} \log E\right)}+\left(A_{X}\right)_{\mathrm{P} 1} E^{-\left(a_{X}+b_{X} \log E\right)}$

$F(E)_{\mathrm{Ip}}=\left(A_{X}\right)_{\mathrm{Ip}} E^{-\left(a_{X}+b_{X} \log E\right)}$

$F(E)_{\mathrm{P} 2}=\left(A_{O}\right)_{\mathrm{P} 2} E^{-\left(a_{O}+b_{O} \log E\right)}+\left(A_{X}\right)_{\mathrm{P} 2} E^{-\left(a_{X}+b_{X} \log E\right)}$.

The parameters to be determined are thus $a_{O}, b_{O}, a_{X}$ and $b_{X}$, besides the normalization factors $\left(A_{O}\right)_{\mathrm{P} 1},\left(A_{O}\right)_{\mathrm{P} 2}$ and $\left(A_{X}\right)_{\mathrm{Ip}}$. The constants $\left(A_{X}\right)_{\mathrm{P} 1}$ and $\left(A_{X}\right)_{\mathrm{P} 2}$ can be obtained from $\left(A_{X}\right)_{\mathrm{Ip}}$ and from the shape $g(f, E)$ of $C_{X}$. Let $F_{X}(E, f)$ be the flux of $C_{X}$ component; we have:

$\frac{\int_{\mathrm{Ip}} F_{X}(E, f) \mathrm{d} f}{\int_{\mathrm{P} 1, \mathrm{P} 2} F_{X}(E, f) \mathrm{d} f}=\frac{\left(A_{X}\right)_{\mathrm{Ip}}}{\left(A_{X}\right)_{\mathrm{P} 1, \mathrm{P} 2}}$.

With the factorization of Eq. (4), we obtain:

$\left(A_{X}\right)_{\mathrm{P} 1, \mathrm{P} 2}=\left(A_{X}\right)_{\mathrm{Ip}} \frac{\int_{\mathrm{P} 1, \mathrm{P} 2} g(f, E) \mathrm{d} f}{\int_{\mathrm{Ip}} g(f, E) \mathrm{d} f}$.

Then, integrating $g(f, E)$ in the proper phase intervals of Ip, P1 and $\mathrm{P} 2$, and obtaining $\left(A_{X}\right)_{\text {Ip }}$ from a spectral fit, we can evaluate $\left(A_{X}\right)_{\mathrm{P} 1}$ and $\left(A_{X}\right)_{\mathrm{P} 2}$.

To obtain a fully consistent picture, in addition to the X-ray data, the spectral distribution of $C_{O}$ must satisfy the optical-UV observations. Sollerman et al. (2000), from STIS/HST observations, found that the UV spectrum of the Crab pulsar is remarkably flat for both peaks and can be described by a single power law, with an energy index $\alpha_{\mathrm{UV}}=-0.11$. To better constrain our

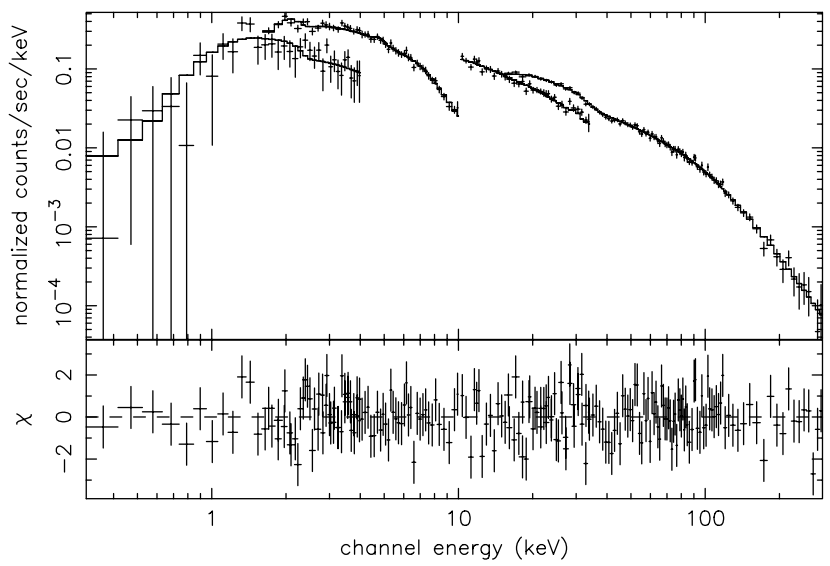

Fig. 7. Spectral best fit of the four BeppoSAX-NFI data sets of Ip with the log-parabolic law of Eq. (9).

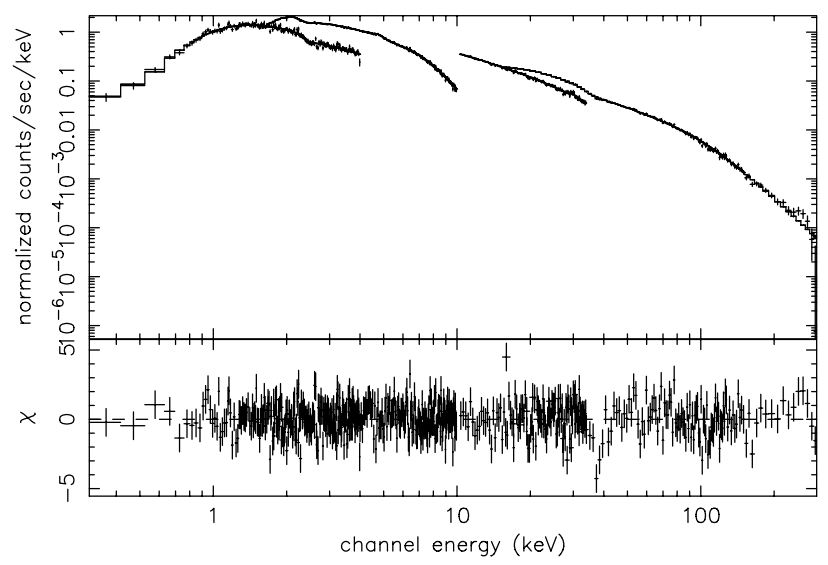

Fig. 8. Spectral best fit of the four BeppoSAX-NFI data sets of P1 with the combined log-parabolic model of Eq. (8) and with the UV constraint of Eq. (13).

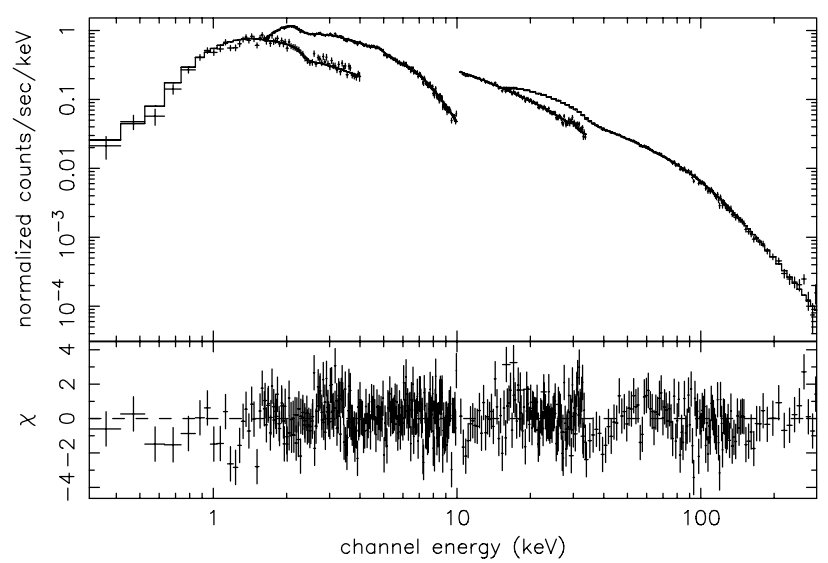

Fig. 9. Spectral best fit of the four BeppoSAX-NFI data sets of $P 2$ with the combined log-parabolic model of Eq. (10) and with the UV constraint of Eq. (13).

spectral parameters we impose the condition that the extrapolation of the $C_{O} \log$-parabolic spectral distribution in the UV range would be consistent with this value and from Eq. (2) we derived the following relation between $a_{O}$ and $b_{O}$ :

$b_{O}=0.21 a_{O}-0.187$

which was included in the spectral best fitting procedure. The resulting best fit spectra with the model of Eqs. (8)-(10) and the 
Table 4. Parameters of the two-component, log-parabolic spectral model fits of BeppoSAX data (LECS, 0.1-4.0 keV; MECS 1.6-10.0 keV; HPGSPC 10-34 keV; PDS 15-300 keV).

\begin{tabular}{cccc}
\hline \hline Parameter & First peak (P1) & Interpeak (Ip) & Second peak (P2) \\
\hline$a_{O}$ & $1.652 \pm 0.003$ & - & $1.670 \pm 0.008$ \\
$b_{O}$ & $0.160 \pm 0.001$ & - & $0.164 \pm 0.001$ \\
$a_{X}$ & - & $1.28 \pm 0.04$ & - \\
$b_{X}$ & - & $0.16 \pm 0.01$ & - \\
$A_{O}\left(^{*}\right)$ & $0.1660 \pm 0.0005$ & - & $(4.72 \pm 0.06) \cdot 10^{-2}$ \\
$A_{X}\left({ }^{*}\right)$ & - & $(2.3 \pm 0.1) \cdot 10^{-2}$ & - \\
$\chi_{r}^{2}$ & $1.02(867$ d.o.f) & $0.99(866$ d.o.f $)$ & $1.09(867$ d.o.f $)$ \\
\hline
\end{tabular}

$\left(^{*}\right)$ photons $\mathrm{cm}^{-2} \mathrm{~s}^{-1} \mathrm{keV}^{-1}$.

residuals for Ip, P1 and P2 of all NFI data sets of BeppoSAX are shown in Figs. 7-9, respectively, and the best fit values are reported in Table 4.

The condition of Eq. (13) should not be considered very stringent in the evaluation of the spectral parameters. In fact, when it is released and only X-ray data are used, the best fit values of $a$ and $b$ are consistent with the ones in in Table 4. Equation (13), therefore, must be considered as a useful tool to reduce the parameter space and to avoid possible inconsistencies between the optical-UV and X-ray data. Moreover, the actual spectral distribution at frequencies much lower than that of the peak can deviate from a pure log-parabola and can approximate a less curved law like a simple power law. Unfortunately, in this frequency range extinction effects are important (see Sect. 3.2) and it is not simple to reach a satisfactory picture.

Three other Crab-like pulsars show curved X-ray spectra that can be fitted by log-parabolae with curvature parameters very similar to that of Crab: PSR B1509-58 $(b=0.16 \pm 0.04$, Cusumano et al. 2001), PSR B0540-69 $(b=0.143 \pm 0.003$, de Plaa et al. 2003) and PSR J0537-6910 ( $b=0.15 \pm 0.05$, Mineo et al. 2004). This finding can be considered an indication that the same radiation mechanism is at work in these sources.

We recall that there are important differences between our modelling and the one of Kuiper et al. (2001). These authors assumed that the pulsed signal observed above $\sim 30 \mathrm{MeV}$, modeled by a single power law, extrapolates down to the X-ray range and therefore fitted the phase resolved spectra by means of this law plus two log-parabolae. The estimate of the curvature parameters is thus affected by the power law, and their values $(0.299$ and 0.084 ) are quite different from those found by us and cannot be directly related to the slopes of the linear best fits of Fig. 3 . The normalization factors of these components are established independently in each phase interval considered, increasing the number of free parameters, whereas we evaluated the normalization factors of $C_{O}$ and $C_{X}$ from their phase distributions, as explained above.

\subsection{The $X$-ray shape and the spectral index of $P 1$}

The two componenent model is founded only on data from the optical to the hard X-ray range and, in principle, it could fail in describing some specific pulsar properties and the spectral behaviour outside this energy range. A useful test is to verify how the model reproduces the characteristics of P1 described in Sect. 5. Figure 4 shows the model profiles computed at the three energies of 2.0, 22 and $85 \mathrm{keV}$, approximately the mean values of the considered energy intervals. The pulse broadening is well described and the only deviation is between the phases 0.025 and 0.075 , where the data show a small excess, which is likely due to the simple analytical shape adopted for $C_{X}$. The search for a better agreement would likely require a more complex formula with a greater number of parameters without a clear improvement of the general description of the pulsed emission.

A simple approximate way to evaluate the mean photon index from the model is to compute the ratio of the summed intensities of $C_{O}$ and $C_{X}$ at the two extremes of the energy range:

$\Gamma_{f}=-\frac{\log \left(F\left(E_{2}\right) / F\left(E_{1}\right)\right)}{\log \left(E_{2} / E_{1}\right)}+\Gamma_{O}$.

Since we used normalised pulse profiles, this spectral index would be zero at the centre of P1 and therefore we added the constants $\Gamma_{O}=1.83$ and 2.14 for the MECS and PDS data, respectively, to match the measured values. In this case the agreement between the observed points and the model, shown in Fig. 5, is generally satisfactory, in particular the asymmetric variation of $\Gamma(f)$ with the softest value at the $\mathrm{P} 1$ centre. Both these results are due to the $C_{X}$ contribution in the phase interval of $\mathrm{P} 1$ necessary to represent the pedestal apparent in the hard-X to lowenergy $\gamma$ rays. These results show that observations are well reproduced if the spectrum of this pedestal is similar to that of Ip, and therefore it is reasonable to consider them all as belonging to the same component.

\subsection{The total pulse profile in the MeV range}

Another important verification of the model is the comparison of pulse profiles and spectra at energies higher than the BeppoSAXNFI range. In particular, we considered the low-energy $\gamma$-ray data obtained by IBIS-PICsIT on board INTEGRAL (Kuiper et al. 2003) and by COMPTEL-CGRO (Kuiper et al. 2001).

We computed the expected pulse shapes from the two component model at the three energies $0.306,0.830$ and $1.90 \mathrm{MeV}$, which are inside the energy ranges of the considered pulse profiles $(0.260-0.364),(0.75-1.0)$ and $(1.0-3.0) \mathrm{MeV}$. We used the spectral parameters of Table 4 and the value of $p$ derived from Eq. (7). The resulting phase histograms are shown in the three panels of Fig. 10 together with the data. We see that the model profiles match well the most important features such as the heights of the two main peaks and gives an acceptable representation of the Ip region. Differences are generally within the observational uncertainties, because of the statistics available in these bands. We see therefore that our model is able to extrapolate well the pulsed emission up to the $\mathrm{MeV}$ range.

\subsection{The emission at optical-UV frequencies}

For a fully consistent description of the Crab pulsed emission we evaluated how the spectral laws derived in the previous Sections extrapolate in the optical-UV range. At these frequencies only the contribution expected from $C_{O}$ is important, that of $C_{X}$ being smaller than one order of magnitude. A simple extrapolation of the model gives a flux lower than that derived by the opticalUV observations by Percival et al. (1993) and Sollerman et al. (2000). As explained in Sect. 3.2, however, reddening effects are very important and even a slight change of the extinction parameter can modify the resulting spectrum.

In order to match the optical points it is not possible to reduce the value of $b$ because it would be inconsistent with the X-ray data. The simplest way is to assume that at frequencies lower than $\sim 0.1 \mathrm{keV}$ the log-parabola tends to approach a power law. A possible spectral distribution, including optical-UV points, is shown in Fig. 11. 


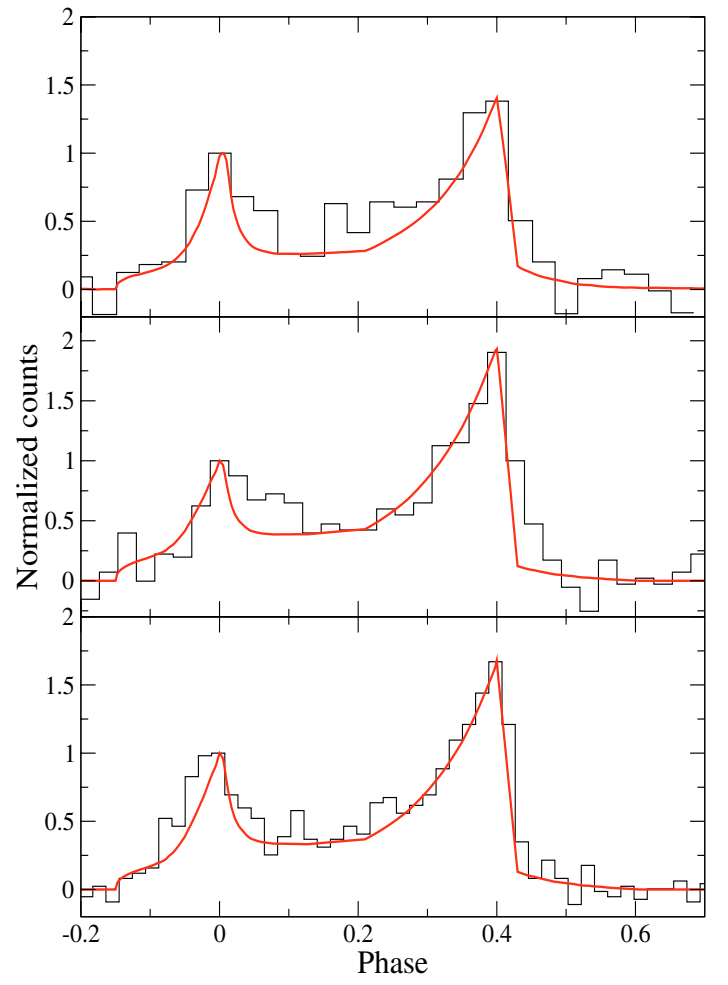

Fig. 10. Comparison between the Crab pulse profiles in the energy ranges 0.260-0.364 (IBIS-PICsIT data from Kuiper et al. 2003, upper panel), $0.75-1.0 \mathrm{MeV}$ and $1.0-3.0 \mathrm{MeV}$ (central and lower panel, COMPTEL-CGRO data from Kuiper et al. 2001) computed using the two component model.

\section{Extension of the two-component model at higher energies: the need for other components}

At energies higher than $\sim 30 \mathrm{MeV}$ the model developed in the previous section based only on the two components $C_{O}$ and $C_{X}$ is not adequate to describe the emission properties of the Crab pulsar. It has been known since early observations that the $\gamma$-ray pulse shape is similar to that of $C_{O}$, although some minor differences are present. The results of COMPTEL and EGRET observations (Kuiper et al. 2001) provided high energy pulse profiles and spectra good enough to develop a more complete model to describe the overall properties of Crab. We recall that there are some similarities between the properties of the pulsed emission in the hard X-rays and in the high energy $\gamma$-rays. Kanbach (1999) showed that the relative flux of P2 to that of P1 increases with energy. The pulse profile above $1 \mathrm{GeV}$ compared to that above $0.5 \mathrm{GeV}$ shows a clear excess of Ip and P2 with respect to P1 (see, e.g., Fig. 12 of Kuiper et al. 2001), Moreover, above $\sim 5 \mathrm{GeV}, \mathrm{P} 2$ seems to be the only dominant feature in the pulse profile, despite the small number of detected events (Thompson 2004).

To explain these properties in a consistent way, from the optical to the GeV band, we extend the previous two component model assuming that there are two other high-energy spectral components, hereafter indicated by $C_{O \gamma}$ and $C_{X \gamma}$, which are strictly related to $C_{O}$ and $C_{X}$, respectively, because their pulse shapes are similar and the spectral distributions are approximated by log-parabolic laws, but shifted in energy. These additional two components imply at least another six adjustable parameters, i.e. the peak energies, curvatures and normalizations

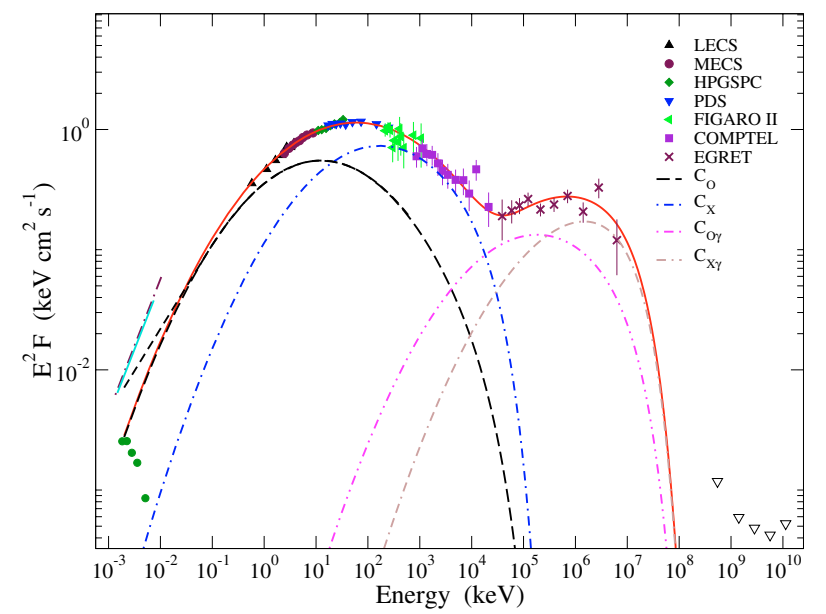

Fig. 11. The broad-band spectral energy distribution of the total averaged pulse of the Crab Pulsar, with the four components of the model. Data points are from BeppoSAX, Compton-CGRO and FIGARO II. We also included $\mathrm{TeV}$ upper limits (downward open triangles) from Aharonian et al. (2004) and the optical data (green circles) from Percival et al. (1993), together with the best fit after reddening corrections (solid cyan line). Also shown (brown dash-dotted line) is the optical dereddened data from Sollerman et al. (2000). A possible low frequency extrapolation of the two component SED to match these optical data is plotted.

of the two log-parabolae, but the statistical quality of the available data does not allow a good estimate of all of them. We assumed, therefore, that their curvatures are equal to that of the corresponding low energy components $(b=0.16)$ and found peak energies and normalization factors able to describe well the observed spectra.

We first adapted this four component model to the total pulsed spectrum and evaluated the best normalization factors of $C_{O \gamma}$ and $C_{X \gamma}$. First we noticed that to be consistent with the upper limits to the pulsed emission in the $\mathrm{TeV}$ range (e.g. Lessard et al. 2000; Aharonian et al. 2004) it was necessary either to increase the values of $b$ or to include an exponential cut-off in the $C_{X \gamma}$ spectrum. We preferred to follow the latter approach, because there is no simple way to fix the curvatures, and found that a cut-off energy $E_{\mathrm{c}}=15 \mathrm{GeV}$ gives a reasonable fit, although this value cannot be precisely evaluated. The same cut-off energy was also assumed for $C_{O \gamma}$, although it was not required by the data, because the spectral steepening of the logparabola reduces the relevance of this component above a few $\mathrm{GeV}$. The resulting spectral energy distribution compared with BeppoSAX, COMPTEL and EGRET data is shown in Fig. 11. We also added some data points obtained from the FIGARO II experiment (Agrinier et al. 1990; Massaro et al. 1998) to fill the gap between $300 \mathrm{keV}$ and $1 \mathrm{MeV}$.

The spectra of P1, Ip, and P2 were computed using the phase intervals of Kuiper et al. (2001) and the intensity ratios derived from the pulse shapes of $C_{O}$ and $C_{X}$. Figures 12-14 show the resulting spectral energy distribution together with the data derived from the literature and our new analysis of BeppoSAX and ISGRI-INTEGRAL data. The agreement with the data in the P1 and $\mathrm{P} 2$ ranges is satisfactory, confirming that in these intervals the pulse shapes of the $C_{O \gamma}$ and $C_{X \gamma}$ components are similar to those of $C_{O}$ and $C_{X}$, respectively, consistent with our assumption. The poor statistics of data in the Ip region does not allow a good enough verification of the model. The SED of Ip has only two components and is poorly known above $10 \mathrm{MeV}$. 


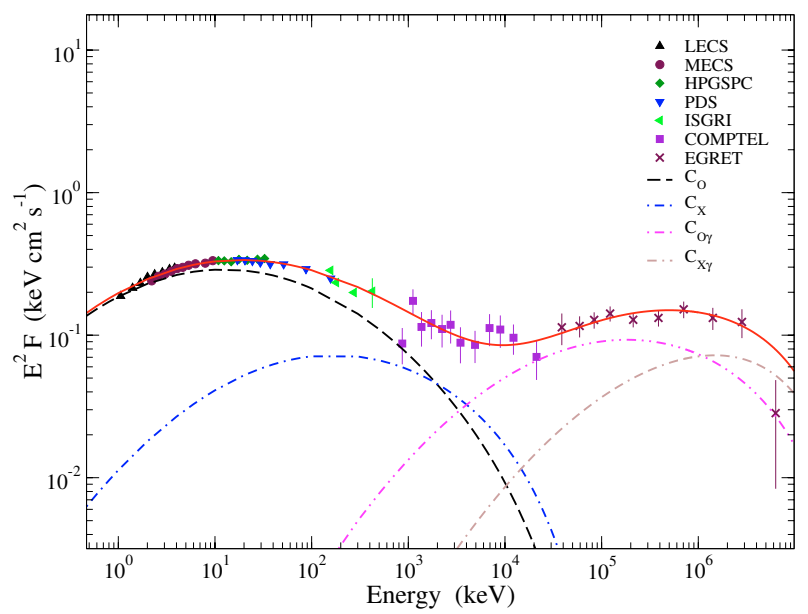

Fig. 12. The broad-band spectral energy distribution of P1 with the four components of the model. Data points are from BeppoSAX, INTEGRAL-ISGRI and Compton-CGRO.

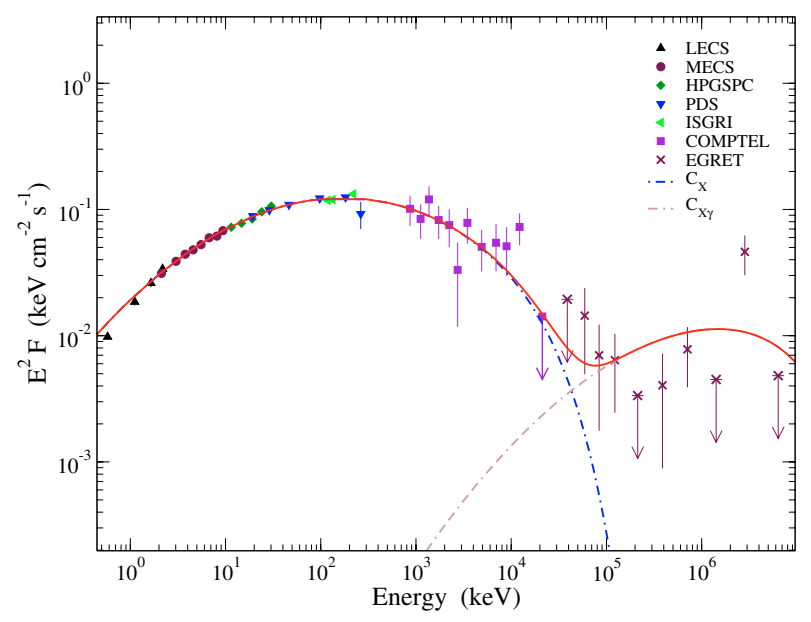

Fig. 13. The broad-band spectral energy distribution of Ip with the components of the model. Data points are from BeppoSAX, INTEGRALISGRI and Compton-CGRO.

However, the model expectation for the Ip intensity was significantly higher than the observational points. Consequently, we reduced the normalization of $C_{X \gamma}$ by a factor of about 2 to approximately match the data. This indicates that the profile of $C_{X \gamma}$ in this range must be different from $C_{X}$, and it does seem to be sharper. When the same change is applied to the spectrum of P1 we did not find a significant modification, the relative contribution of $C_{X \gamma}$ being much lower than $C_{O \gamma}$. Note in the SEDs of $\mathrm{P} 1$ and $\mathrm{P} 2$ how the mild spectral curvature is well reproduced by the superposition of the four components and that the positions of the minima are not the same: it is around $10 \mathrm{MeV}$ for $\mathrm{P} 1$ and around $40 \mathrm{MeV}$ for P2. At present, however, the data do not allow an accurate estimate of this difference. New observations with richer statistics will be useful to constrain this feature more precisely, to provide further support for our multicomponent model and to evaluate the component parameters. All the main parameters of the full model are given in Table 5.

This model is not univocally determined by data. It is apparent from Fig. 12 that in COMPTEL and EGRET data the P1 spectrum can be fitted by a single power law with the only exception being the point at the highest energy where a cut-off may be present, as already proposed by Kuiper et al. (2001).

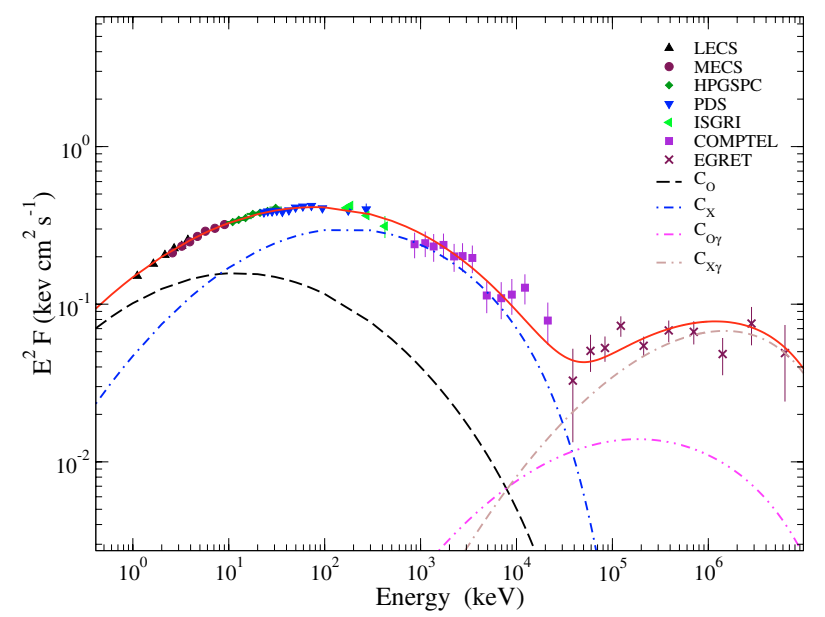

Fig. 14. The broad-band spectral energy distribution of $\mathrm{P} 2$ with the four components of the model. Data points are from BeppoSAX, INTEGRAL-ISGRI and Compton-CGRO.

Table 5. Main parameters of the four component model of the pulsed emission of the Crab pulsar.

\begin{tabular}{cccc}
\hline \hline Component & $\begin{array}{c}E_{\mathrm{p}} \\
\mathrm{MeV}\end{array}$ & $\begin{array}{c}E_{\mathrm{c}} \\
\mathrm{GeV}\end{array}$ & $\begin{array}{c}\left.L_{\mathrm{bol}}{ }^{*}\right) \\
\mathrm{erg} / \mathrm{s} 10^{36}\end{array}$ \\
\hline$C_{O}$ & $12.2 \times 10^{-3}$ & - & 2.8 \\
$C_{X}$ & $178 \times 10^{-3}$ & - & 3.8 \\
$C_{O \gamma}$ & 300 & $\leq 15$ & 0.97 \\
$C_{X \gamma}$ & 2000 & $\sim 15$ & 0.92 \\
\hline
\end{tabular}

$\left(^{*}\right)$ Assuming isotropic emission $\left(\omega_{a}=4 \pi\right)$.

In the spectral plots evaluated by these authors assuming a power law component with a phase independent spectral index, one can see that EGRET data for P1 are all in excess with respect to the best fit, while practically all those of COMPTEL lie under it. Moreover, large systematic deviations from this best fit are apparent in the leading and trailing wings of P1 and P2 (see also Fierro et al. 1998). We conclude that the model based only on components having spectral distributions approximated by a logparabolic law gives a simpler picture of this complex spectral behaviour.

Another test of the model is to verify how it reproduces the flux ratios $\mathrm{P} 2 / \mathrm{P} 1$ and $\mathrm{Ip} / \mathrm{P} 1$, frequently considered in the literature to describe the pulsed emission of Crab. We computed these ratios for the phase intervals adopted by Kuiper et al. (2001), and the results are plotted in Figs. 15 and 16 for $\mathrm{P} 2 / \mathrm{P} 1$ and $\mathrm{Ip} / \mathrm{P} 1$, respectively. The general behaviours of both ratios are well reproduced, in particular the sharp decreases between 1 and $10 \mathrm{MeV}$ and the following slow increase to the $\mathrm{GeV}$ range. We stress that the sharpness of the decrease depends on the curvature and peak energies of log-parabolic spectra and of the adopted cut-off.

We also calculated the bolometric flux of each component by integrating the log-parabolic spectral distribution over all energies. This integral can be easily computed analytically (Massaro et al. 2004) and the result is:

$F_{\text {bol }}=\int_{0}^{\infty} A E^{-(a+b \log E)} \mathrm{d} E=\frac{2.70}{\sqrt{b}} E_{\mathrm{p}}^{2} F\left(E_{\mathrm{p}}\right)$.

The luminosity of each of the four components is $L=F_{\text {bol }} \times$ $d^{2} \omega_{a}$, where $d$ is the distance to the Crab and $\omega_{a}$ is the solid angle occupied by the emission beam. We take $d \simeq 2 \mathrm{kpc}=6.2 \times$ $10^{21} \mathrm{~cm}$, whereas the value of $\omega_{a} \leq 4 \pi$ is difficult to estimate 


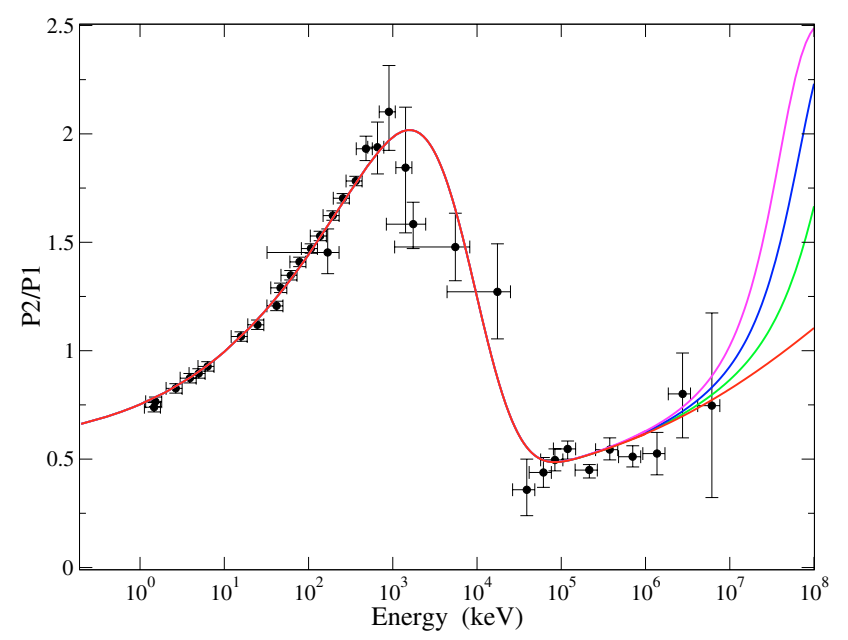

Fig. 15. The ratio between the fluxes of $\mathrm{P} 2$ and $\mathrm{P} 1$ phase regions $(\mathrm{P} 1$ : $-0.06-0.04$; P2: 0.32-0.43), compared to the predictions of the model. The data points come from various experiments (Kuiper et al. 2001). The various extrapolations above $1 \mathrm{GeV}$ correspond to different values of the cut-off energy of the $C_{O \gamma}$ spectrum: $15 \mathrm{GeV}$ (red), $13 \mathrm{GeV}$ (green), $11 \mathrm{GeV}$ (blue) and $9 \mathrm{GeV}$ (violet) - from bottom to top.

because it depends on the emission geometry, which is not completely known. The resulting values for the isotropic case $\left(\omega_{a}=4 \pi\right)$ are given in Table 5, but they are upper limits to the actual values. The total bolometric luminosity, obtained by adding the contributions of the four components, is $L=8.5 \times 10^{36} \mathrm{erg}$ $\mathrm{s}^{-1}$, which drops to $\sim 6.8 \times 10^{35} \mathrm{erg} \mathrm{s}^{-1}$ for $\omega_{a}=1 \mathrm{str}$.

\section{Discussion}

The development of a detailed physical model for the spectral and phase distributions of the broad-band emission from the $\mathrm{Crab}$ pulsar is a difficult problem. It requires a precise geometrical definition of the regions inside the magnetosphere where the observed radiation originates, and the knowledge of parameters like the orientation angles between the magnetic axis and the line of sight to the spin direction. Several models have appeared in the literature based on either polar cap or outer gap geometries. Usually, these models are focused on reproducing either the total spectrum or the phase profile and generally they are not fully satisfactory in explaining the complex observational picture. Moreover, the possibility that the observed features of the pulsed signal can arise from the superposition of two or more distinct components is not taken into account.

We followed another approach and searched for a possible interpretation of the Crab signal based on the superposition of two or more components that provides a consistent description of the spectral and phase distributions. MCLM showed that a possible explanation of the energy dependence of the pulse shape of $\mathrm{Crab}$ in the soft to hard X-rays is that we are observing two emission components with different phase and energy distributions. In that paper we introduced an empiric model, based on a collection of BeppoSAX observations, covering the energy range from 0.1 to about $300 \mathrm{keV}$, from which it was possible to estimate some properties of the main components. Moreover, we showed that the X-ray spectrum of $\mathrm{P} 1$ presents a mild curvature well fitted by a log-parabola. At energies higher than $30 \mathrm{MeV}$, however, this two component model fails to represent both pulse profiles and spectra, as observed by EGRET-CGRO (Fierro et al. 1998). To take into account $\gamma$-ray data, Kuiper et al. (2001) proposed the existence of three components, two of them having log-parabolic spectra, while the third one with a power law spectrum and a phase modulated intensity which reaches the highest level in correspondence of the two peaks and is almost absent in the Ip region. According to our point of view, however, this hypothesis is not fully consistent with the data, because the flux increases of Ip and P2 are very similar, suggesting that their $\mathrm{X}$-ray emission is dominated by the same physical mechanism. Moreover, the parameters describing the curvatures of their two log-parabolic distributions are very different, while the analysis of X-ray spectra reported in Sect. 6.2 indicates that the curvature is rather stable with phase at an intermediate value between them. Finally, the power-law component does not match well the data in some phase intervals at $\gamma$-ray energies where its contribution is dominant.

To achieve a more consistent scenario, in the present paper we propose a model of the pulsed emission from Crab based on four components. It is properly a two double-component model because each component pair has similar phase distributions and spectra shifted in energy. At present, our model gives an empiric description of the broad-band properties and its validity is based on the limited number of assumptions and on the resulting capability to obtain a consistent description of the observations outside the energy ranges used to evaluate the parameters. For instance, the model extrapolates well the pulse profiles in the $\mathrm{MeV}$ range (see Sect. 6.4) and the change with energy of the P2/P1 and $\mathrm{Ip} / \mathrm{P} 1$ ratios. We stress that $\mathrm{Crab}$ is not the only pulsar that shows a behaviour that can be interpreted by a multicomponent model. Harding et al. (2002) proposed that the X-ray pulsed emission from Vela originates from two non-thermal components, one coincindent in phase with the $\gamma$-ray pulse profile and the other one with the optical.

The physical processes at the origin of these components, and the location in the magnetosphere where they occur, must be further investigated. The development of a detailed physical model of the high energy emission in the Crab magnetosphere is beyond the aim of the present paper, however some general indications on it can be derived from our conclusions. The same phase distribution assumed for each pair of components, such as $C_{O}$ and $C_{O \gamma}$, suggests that their angular pattern and emission sites must be coincident or very close, otherwise the aberration effects would modify the pulse shapes. However, this is not necessarily true because on the trailing last open field line, aberration and propagation time effects would cancel to form a caustic, as shown by Dyks \& Rudak (2003).

According to a widely accepted scenario, primary electrons accelerated in a magnetospheric gap emit high energy photons which produce $e^{ \pm}$pairs against the magnetic field. These secondary particles emit synchrotron radiation in the optical to $\mathrm{MeV}$ energy range. A first possibility to be considered is that $C_{O}$ and $C_{X}$ are synchrotron radiation from two different places whereas the corresponding high energy components are inverse Compton upscattered photons. A very early inverse Compton model for Crab was proposed by Zheleznyakov \& Shaposhnikov (1972), when the $\gamma$-ray emission properties were known very poorly, and another model was applied to the Vela pulsar by Morini (1983). A more recent development is that of Cheng \& Wei (1995). Their model is based on the outer gap geometry and assumes that optical to hard X-ray photons are emitted by secondary $e^{ \pm}$pairs created outside the accelerating region by high-energy primary curvature photons. $\gamma$-rays are then emitted via a synchrotron selfCompton (SSC) process. In this case we expect that Compton scattering of hard X-ray photons occurs mainly in the KleinNishina regime, and therefore the observed high energy cut-off gives an estimate of the maximum energy of electrons. The fact 


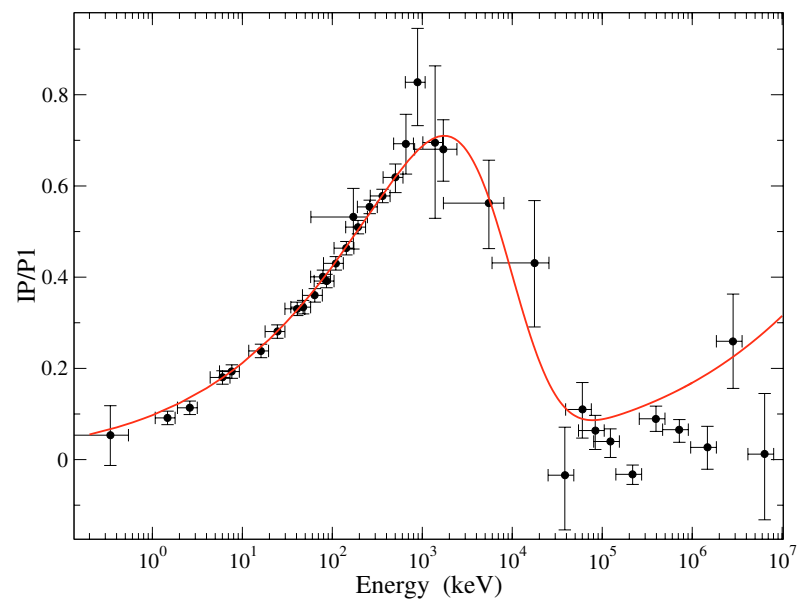

Fig. 16. The ratio between the fluxes of $\mathrm{Ip}$ and $\mathrm{P} 1$ phase regions (P1: $-0.06-0.04$; Ip: $0.14-0.25)$, compared to the predictions of the model. The data points come from various experiments (Kuiper et al. 2001).

that it is in the $\mathrm{GeV}$ range could be consistent with their origin from magnetic pair production.

There is, however, another possibility for the origin of the high energy components. In a polar cap scenario these photons could be those emitted by primary electrons via curvature radiation and not absorbed by magnetic pair production throughout the magnetosphere. The attenuation length for this process can be approximated by (Erber 1966):

$x\left(B_{\perp}, E_{\gamma}\right)=2.3 \times 10^{-8} \frac{B_{\mathrm{cr}}}{B_{\perp}} \exp \left[\frac{4 B_{\mathrm{cr}}}{3 B_{\perp}} \frac{2 m c^{2}}{E_{\gamma}}\right] \mathrm{cm}$,

where $B_{\perp}$ is the transverse magnetic field seen by a photon of energy $E_{\gamma}$, and $B_{\mathrm{cr}}=4.414 \times 10^{13}$ is the quantum critical field. It is easy to verify that a mean free path of the order of $10^{4} \mathrm{~cm}$ for $5 \mathrm{GeV}$ photons is obtained in a transverse field $B_{\perp} \simeq 7.5 \times 10^{8} \mathrm{G}$ and that it increases very rapidly even for decreasing $E_{\gamma}$. This cut-off in the curvature $\gamma$-ray spectra at $\mathrm{GeV}$ energies has been verified since early numerical calculations, including an accurate evaluation of the absorption coefficient, for a polar cap acceleration (Salvati \& Massaro 1978; Massaro \& Salvati 1979).

Although our multi-component model gives a consistent picture of the pulsed emission from Crab it is not yet completely determined by observational data. An important test will be the study of the spectra and pulse profiles at energies higher than a few $\mathrm{GeV}$ : in particular, we expect that above about $5 \mathrm{GeV}, \mathrm{P} 2$ would be the dominant feature, while a good measure of the flux in the Ip region will be very useful to draw the phase structure of $C_{X \gamma}$. High quality data in this energy range will be obtained with the LAT telescope on board the GLAST mission to be operative next year. Another interesting test of the model could be obtained from phase resolved polarization measures in the $\mathrm{X}$-ray range. We know that the optical linear polarization of the Ip differs from those of P1 and P2 both in strength and direction (Smith et al. 1988; Kanbach et al. 2003). According to our model the polarization of the $\mathrm{P} 2 \mathrm{X}$-ray emission must be more similar to that of Ip, because of the higher contribution of $C_{X}$. New generation high sensitivity polarimetry for X-ray astronomy, such as that proposed by Costa et al. (2001), could be very useful. Finally, the observation of other young spin powered pulsars, if their high energy emission is similar to Crab, could also be very useful because we expect to observe them from different directions and therefore to see other pulse shapes, depending on the various combinations of the two components.
Acknowledgements. We are grateful to M. Salvati for interesting comments and to L. Kuiper who kindly gave us COMPTEL and EGRET data. This work has been partially supported by Università di Roma "La Sapienza".

\section{References}

Adler, S. L. 1971, Ann. Phys., 67, 599

Agrinier, B., Masnou, J. L., Parlier, B., et al. 1990, ApJ, 355, 645 Aharonian, F., Akhperjanian, A., Beiliche, M., et al. 2004, ApJ, 614, 897 Anders, E., \& Ebihara, M. 1982, Geochim. Cosmochim. Acta, 46, 2363 Anders, E., \& Grevesse, N. 1989, Geochim. Cosmochim. Acta, 53, 197 Balucinska-Church, M., \& McCammon, D. 1992, ApJ, 400, 699 Bednarek, W., Cremonesi, O., Treves, A., et al. 1992, ApJ, 390, 489 Cheng, K. S., Ho, C., \& Ruderman, M. A. 1986a, ApJ, 300, 500 Cheng, K. S., Ho, C., \& Ruderman, M. A. 1986b, ApJ, 300, 522 Cheng, K. S., Ruderman, M. A., \& Zhang, L. 2000, ApJ, 537, 964 Chiang, J., \& Romani, R. W. 1994, ApJ, 436, 754

Costa, E., Soffitta, P., Bellazzini, R., et al. 2001, Nature, 411, 662

Cusumano, G., Mineo, T., Massaro, E., et al. 2001, A\&A, 375, 397

Daugherty, J. K., \& Harding, A. K. 1994, ApJ, 429, 325

Daugherty, J. K., \& Harding, A. K. 1996, ApJ, 458, 278

de Plaa, J., Kuiper, L., \& Hermsen, W. 2003, A\&A, 400, 1013

Dyks, J., \& Rudak, B. 2003, ApJ, 598, 1201

Erber, T. 1966, Rev. Mod. Phys., 38, 626

Fierro, J. M., Michelson, P. F., Nolan, P. L., et al. 1998, ApJ, 494, 734

Fiore, F., Guainazzi, M., \& Grandi, P. 1999, in Cookbook for BeppoSAX NFI Spectral Analysis, BeppoSAX Science Data Center, version 1.2

Fukuzawa, Y., Ishida, M., \& Ebisawa, K. 1997, ASCA News, 5, 1

Harding, A. K., Strickman, M. S., Gwinn, C., et al. 2002, ApJ, 576, 376

Hirotani, K., Harding, A. K., \& Shibata, S. 2003, ApJ, 588, 430

Jahoda, H. K. 1994, A\&AS, 26, 894

Kanbach, G. 1999, Proc. 3rd INTEGRAL Workshop, Astroph. Lett. Comm., 38, 17

Kanbach, G., Kellner, S., Schrey, F., et al. 2003, Proc. SPIE, 4841, 82

Kuiper, L., Hermsen, W., Cusumano, G., et al. 2001, A\&A, 378, 918

Kuiper, L., Hermsen, W., Walter, R., \& Foschini, L. 2003, A\&A, 411, L31

Lebrun, F., Leray, J. P., Lavocat, P., et al. 2003, A\&A, 411, L141

Lessard, R. W., Bond, I. H., Bradbury, S. M., et al. 2000, ApJ, 531, 942

Massaro, E., \& Salvati, M. 1979, A\&A, 71, 51

Massaro, E., Feroci, M., Costa, E., et al. 1998, A\&A, 338, 184

Massaro, E., Cusumano, G., Litterio, M., \& Mineo, T. 2000, A\&A, 361, 695 (MCLM)

Massaro, E., Perri, M., Giommi, P., \& Nesci, R. 2004, A\&A, 413, 489

Mineo, T., Cusumano, G., Segreto, A., et al. 1997, A\&A, 327, 21

Mineo, T., Cusumano, G., \& Massaro, E. 2004, Nucl. Phys. B Proc. Suppl., 132, 632

Mineo, T., Ferrigno, C., Foschini, L., et al. 2006, A\&A, 450, 617

Morini, M. 1983, MNRAS, 202, 495

Morrison, R., \& McCammon, D. 1983, APJ, 270, 119

Much, R., Bennett, K., Buccheri, R., et al. 1995, A\&A, 299, 435

Muslimov, A. G., \& Harding, A. K. 2003, ApJ, 588, 430

Percival, J. W., Biggs, J. D., Dolan, J. F., et al. 1993, ApJ, 407, 276

Pravdo, S. H., \& Serlemitsos, P. J. 1981, ApJ, 246, 484

Pravdo, S. H., Angelini, L., \& Harding, A. K. 1997, ApJ, 491, 808

Predehl, P., \& Schmitt, J. H. M. M. 1995, A\&A, 293, 899

Ride, S. K., \& Walker, A. B. C. 1977, A\&A, 61, 347

Romani, R. K. W., \& Yadigaroglu, I.-A. 1995, ApJ, 438, 314

Rots, A. H., Jahoda, K., \& Lyne, A. G. 2004, ApJ, 605, L129

Salvati, M., \& Massaro, E. 1978, A\&A, 67, 55

Smith, F. G., Jones, D. H. P., Disck, J. S. B., et al. 1988, MNRAS, 233, 305

Sollerman, J., Lundqvist, P., Lindler, D., et al. 2000, ApJ, 537, 861

Sturner, S. J., \& Dermer, C. D. 1994, ApJ, 420, L79

Staelin, D. H., \& Reifenstein, E. C. 1968, Science, 162, 1481

Tennant, A. F., Becker, W., Juda, M., et al. 2001, ApJ, 554, L173

Thompson, D. J. 2004, Proc. Cosmic Gamma-ray Sources, ed. K. S. Cheng, \& G. E. Romero, ASSL (Kluwer Acad Publ.), 304, 149 [arXiv:astro-ph/0312272]

Toor, A., \& Seward, F. D. 1977, ApJ, 216, 560

Vivekanand, M. 2002, A\&A, 391, 1033

Weisskopf, M. C., O’Dell, S. L., \& Paerels, F., et al. 2004, ApJ, 601, 1050

Willingale, R., Aschenbach, B., Griffiths, R. G., et al. 2001, A\&A, 365, L212

Wilms, J., Allen, A., \& McCray, R. 2000, ApJ, 542, 914

Zhang, L., \& Cheng, K. S. 2002, ApJ, 569, 872 